\title{
Effect of location of eggs in the incubator on hatchability of eggs from Bronze turkey breeders of different ages
}

\author{
M. Özçelik ${ }^{1 \#}$, F. Ekmen ${ }^{2}$ and Ö. Elmaz ${ }^{1}$ \\ ${ }^{1}$ Mehmet Akif Ersoy University, Faculty of Veterinary Medicine, Department of Animal Breeding, 15100, \\ Burdur, Turkey \\ ${ }^{2}$ Harran University, Faculty of Veterinary, Department of Animal Husbandry, Urfa-Turkey
}

\begin{abstract}
The objective of this study was to determine the effect of location in an incubator on the hatching characteristics of eggs obtained from Bronze turkeys of different ages, viz. at the ages of 8,10 and 12 months. In the study 330 eggs were placed in each of six locations within an incubator, totalling 1980 eggs from each age group, and 5940 eggs overall. The highest fertility rate and hatchability of all eggs set, and of fertile eggs were recorded in the eggs from 10-month old breeders. The lowest rates of shell adhesion deaths, null poults and poults with abnormalities were also observed in this group. In all age groups investigated, the hatchability of all eggs set and of fertile eggs was the highest in eggs placed in the front of the machine. Furthermore, early and middle stage deaths during incubation were lower in the front of the incubator compared to the back. The differences between eggs placed in the upper, middle and lower parts of the machine with respect to the investigated characteristics were not statistically significant. Although these results were obtained from a specific incubator, the locations inside and all applications (temperature, humidity, shaking, etc.) were within accepted standards. Therefore, results are applicable, in general.
\end{abstract}

Keywords: Egg location, hatchability, incubation, egg size, turkey

${ }^{\#}$ Corresponding author. E-mail: mozcelik@mehmetakif.edu.tr

\section{Introduction}

One of the main objectives in turkey breeder production is to increase the number of poults produced. The production cost of poults can be lowered by increasing egg yield, fertilization capacity and hatchability. Egg yields in turkeys are lower than that of other poultry species. For this reason the primary means of increasing the number of poults is to increase the number of eggs for hatching produced by each turkey, and to subject these eggs to the most effective hatching procedures available (Camc1 \& Sarıca, 1991; Kaygisız, 1993). In addition to low egg yield, unsatisfactory egg fertility and hatchability constitute a major problem for breeding enterprises. Therefore, Turkish breeding programmes are searching for ways of determining the factors affecting hatchability, and for defining and improving the environmental factors affecting hatchability (Kaygisız, 1993).

Important advances have been achieved in hatching features and poult quality, and more importantly, in fertility as a result of the increase in the efficiency of breeder animals and the development of husbandry conditions, the improvement in incubators and the attention paid to health prevention measures. Furthermore, hatching has been a continuously developing business in the poultry breeding enterprise, with its importance increasing.

However, these developments have been accompanied by some problems. One of the most important is the decrease in hatchability due to the increased weight of the eggs (Elibol et al., 2000). It has been reported that the hatchability of medium sized turkey eggs is better than that of small or large eggs (Brunson \& Godfrey, 1953; Kaygisiz et al., 1994). It has been determined that heavy eggs have a higher last-phasedeath-rate in broilers than lighter eggs (Reinhart \& Hurnik, 1984; Hagger et al., 1986; Ogunshile \& Sparks, 1995). The same phenomenon has been observed in turkey eggs (Reinhart \& Moran, 1979).

Developments in the hatching sector have created the necessity that the capacity of incubators be increased (Deeming, 1996). This has directed the attention to the providing of homogenous air circulation as well as heat distribution among the eggs. Mauldin \& Buhr (1995a) reported that in incubators with multiple entrances there is approximately a $10^{\circ} \mathrm{C}$ difference in temperature between the surface of the egg and that displayed by the machine, and this may negatively affect the hatchability of fertile eggs. 
It has been observed that the location of eggs in an incubator (front, back, upper, middle, and lower) does not have a significant effect on embryo deaths, but that poults hatch earlier in eggs located on the upper part of the incubator than in other sections. This condition suggests that the temperature is higher in the upper part of the machine than elsewhere, and thus that the temperature in the machine may not be uniform, and should be better controlled (Reinhart \& Hurnik, 1984). The increase in the weight of eggs due to the selecting for this trait causes the embryos to produce more heat, and this results in an increase in last-phaseembryonic-deaths related to high temperature stress (Sotherland et al., 1987; Tullett, 1990; French, 1994a; b; Deeming, 1996; French, 1997). French (1994b) reported that a decrease in temperature from $37.5^{\circ} \mathrm{C}$ to $36.5^{\circ} \mathrm{C}$ improved the hatchability of large fertile turkey eggs, but that the same effect was not observed in small eggs.

Hatching performance in commercial hatcheries may be improved by modifying the hatching conditions to meet the requirements of individual batches of eggs. A brief review of available evidence that such a strategy can be used to improve results highlighted the paucity of data available. In large hatcheries a more simplistic approach may be required, namely, by batching of eggs according to the age of the breeder flock. Further technological advances in incubator design may be required before it may be feasible to adjust incubation conditions to meet the requirements of each batch of eggs (French, 2002).

The present study was conducted to determine the effect of the position of eggs obtained from a Bronze turkey flock at three different ages in the incubator and hatcher on hatching performance. In the study the limitation of air circulation in artificial incubators and the importance of the speed of air, egg temperature and metabolism of the developing embryo were evaluated.

\section{Materials and Methods}

The eggs for this study were collected from a Bronze turkey breeder flock in the Bingöl Apiculture and Turkey Production Station, and incubated at the same location. Eggs were collected from the same breeder flock at the ages of 8,10 and 12 months. The flock was raised in an intensive system in basesupported poultry houses. Free mating was used in the flock, and the ratio of males to females was $1: 10$. No systematic selection was applied in the flock.

Three hundred and thirty eggs were placed in each of six positions in an incubator, thus using 1980 eggs $(330 \times 6)$ in each age group, and a total of $5940(1980 \times 3)$ eggs. A fully automatic setter with a capacity of 26000 eggs and a separate hatcher were used. Eggs from the three age groups $(8,10$ and 12 months) and six setter positions (front or back x upper, middle or lower) were used. For each age group, 3\% of the eggs (60 eggs) were weighed and mean egg weights were determined prior to setting. All eggs were stored under the same conditions for seven days before being set. The hatching process was replicated three times for each age with 330 eggs. The eggs were randomly divided into six groups before being placed in the setter. The position of the eggs in the hatcher was the same as that in the setter. Fumigation for disinfection was applied to eggs and machines, using potassium permanganate, while $40 \%$ formaldehyde was used during the storage period in the setter and in the hatcher. The processes utilized; fumigation, storage conditions and machine conditions were uniform for each hatching period.

Temperature and relative humidity in the incubator were $36.7-37.8^{\circ} \mathrm{C}$ and $55-60 \%$, respectively; and in the hatcher, $35.6-36.7^{\circ} \mathrm{C}$ and $75-80 \%$, respectively. On the $14^{\text {th }}$ day of incubation the eggs were candled to determine fertility. Embryonic deaths were determined on the 25 th day by candling when the eggs were transferred to the hatcher. Hatching began on day 27 and was completed by the end of day 28. At the end of every hatching process, the number of infertile eggs, hatched healthy eggs, surplus poult (null eggs,) poults with abnormalities and unhatched eggs were counted and separated. The unhatched eggs were opened and the number of infertile and contaminated (infected) eggs was determined; and among the fertile eggs, the early phase $(0-12 \mathrm{~d})$, middle phase $(13-24 \mathrm{~d})$, late phase $(25-28 \mathrm{~d})$ and shell adhesion (pierced the shell and dried, or hatched but adhered to the shell) death rates were determined. Using these data, the variables investigated in the study were determined as follows (Erensayın, 2000):

Fertility rate $(\%)=($ Number of fertile eggs / Total number of eggs set $) \times 100$;

Hatchability of all eggs $(\%)=($ Number of poults hatched / Total number of eggs set $) \times 100$;

Hatchability of fertile eggs $(\%)=($ Number of poults hatched / Number of fertile eggs $) \times 100$;

Surplus poult rate $(\%)=($ Number of surplus poults / Number of fertile eggs $) \times 100$;

Abnormality rate $(\%)=($ Number of poults with abnormalities / Total number of poults hatched $) \times 100$; 
Early-phase-deaths $(\%)=($ Number of embryos dying in the early phase / Number of unhatched eggs) $\mathrm{x}$ 100 ;

Middle-phase-deaths $(\%)=($ Number of embryos dying in the middle phase $/$ Number of unhatched eggs) x 100 ;

Late-phase-deaths $(\%)=($ Number of embryos dying in the late phase / Number of unhatched eggs) $\mathrm{x}$ 100

Shell adhesion deaths $(\%)=($ Number of embryos dying adhered to shell / Number of unhatched eggs $)$ $\mathrm{x} 100$.

A factorial experimental design with three factors (age, location and sub-location) was used. The statistical evaluation was done using Univariate Variance Analysis of the General Linear Model in concordance with this arrangement. In the determination of different groups, Duncan's multiple comparison method was utilized. Also, the Independent Sample t-test was used in the determination of the differences between the location groups (front and back) for each age group; and Kruskal Wallis test was used in the determination of the differences between the location sub-groups (Jerrold, 1996). The calculations were made using the SPSS programme package (SPSS, 2002).

\section{Results and Discussion}

Mean values of the hatching results as affected by age and location, and statistical evaluations are presented in Table 1. The effect of age was found to be significant for fertility rate, hatchability of all eggs set and of fertile eggs $(\mathrm{P}<0.001)$, shell adhesion deaths $(\mathrm{P}<0.01)$ and abnormality rate $(\mathrm{P}<0.05)$. Furthermore, the differences in early-phase-deaths, shell adhesion deaths, hatchability of all eggs set, hatchability of fertile eggs $(\mathrm{P}<0.001)$ and the surplus poult $(\mathrm{P}<0.01)$ were significant for the eggs placed in the front vs. the back parts of the incubator. The differences in fertility rate were significant only for eggs placed in the upper, middle and lower parts of the incubator $(\mathrm{P}<0.05)$.

Age of the breeder flock was an important factor affecting the weight of the egg, internal and external quality characteristics of the egg, hatching results and the quality of the poult. As hen age increases, the weight of the egg increases and both shell quality and internal egg quality decrease (Erensayın, 2000). Many investigators have reported that the weight of the egg influences hatching results (Karaçanta et al., 1977; Reinhart \& Hurnik, 1984; Wilson, 1991; Kaygisız et al., 1994). It has been reported that hatchability of turkey eggs is maximized with eggs weighing between 70 - $85.5 \mathrm{~g}$ (Karaçanta et al., 1977; Erişir, 1999).

In this study mean egg weight was $67.4 \mathrm{~g}$ in the 8-months group, $70.3 \mathrm{~g}$ in the 10 -months group, and $70.1 \mathrm{~g}$ in the 12-months group. The highest fertility, hatchability of all eggs set and hatchability of fertile eggs were recorded in the 10-months age group. Moreover, the lowest rates of shell adhesion death, null poults and poults with abnormalities were observed in this group.

Hatching features were investigated separately in the different age groups. Means related to the hatching features as affected by the different locations, and the results of the statistical evaluation are shown in Table 2 for the 8-month age group, in Table 3 for the 10-month group and in Table 4 for the 12-month age group.

When the differences between the front and back layout arrangements are considered, only the hatchability was found to be significant $(\mathrm{P}<0.05)$ in the eggs obtained from the 8-month age group. Differences in early-phase-deaths, hatchability of fertile eggs, shell adhesion deaths, hatchability of all eggs set and surplus poult were significant in the eggs from the 10 -month age group $(\mathrm{P}<0.05)$, as seen in Table 3; and differences in early-phase-deaths, hatchability of fertile eggs $(\mathrm{P}<0.05)$ and hatchability of all eggs set $(\mathrm{P}<0.01)$ were significant in the eggs from the 12-month age group (Tables $2-4)$. However, none of the differences between the upper, middle and lower layout arrangement groups were found to be statistically significant in any of the three age groups. 
Table 1 Main effects of age and egg location groups on fertility, embryonic mortality, hatchability and abnormalities of turkey eggs

\begin{tabular}{|c|c|c|c|c|c|c|c|c|c|c|c|c|c|c|c|c|c|c|c|}
\hline \multirow{3}{*}{ Features } & \multirow{3}{*}{$\mathrm{n}$} & \multirow{2}{*}{\multicolumn{2}{|c|}{$\begin{array}{c}\text { Fertility } \\
\text { rate } \\
(\%)\end{array}$}} & \multicolumn{8}{|c|}{ Deaths $(\%)$} & \multirow{2}{*}{\multicolumn{2}{|c|}{$\begin{array}{c}\text { Hatchability } \\
\text { of all eggs } \\
(\%)\end{array}$}} & \multirow{2}{*}{\multicolumn{2}{|c|}{$\begin{array}{c}\text { Hatchability of } \\
\text { fertile eggs } \\
(\%)\end{array}$}} & \multirow{2}{*}{\multicolumn{2}{|c|}{$\begin{array}{l}\text { Surplus poult } \\
\text { rate } \\
(\%) \\
\end{array}$}} & \multirow{2}{*}{\multicolumn{2}{|c|}{$\begin{array}{l}\text { Abnormality } \\
\text { rate } \\
(\%)\end{array}$}} \\
\hline & & & & \multicolumn{2}{|c|}{ Early-phase } & \multicolumn{2}{|c|}{ Middle-phase } & \multicolumn{2}{|c|}{ Late-phase } & \multicolumn{2}{|c|}{ Shell adhesion } & & & & & & & & \\
\hline & & mean & s.e. & mean & s.e. & mean & s.e. & mean & s.e. & mean & s.e. & mean & s.e. & mean & s.e. & mean & s.e. & mean & s.e. \\
\hline \multicolumn{20}{|l|}{ Age } \\
\hline 8 months & 1980 & $85.8^{\mathrm{b}}$ & 0.49 & 49.6 & 1.91 & 6.3 & 0.85 & 0.0 & 0.18 & $20.0^{\mathrm{a}}$ & 1.22 & $37.4^{\mathrm{b}}$ & 0.77 & $43.6^{\mathrm{b}}$ & 0.882 & 4.4 & 0.83 & $3.5^{\mathrm{a}}$ & 0.73 \\
\hline 10 months & 1980 & $90.6^{\mathrm{a}}$ & 0.49 & 52.9 & 1.91 & 7.6 & 0.85 & 0.4 & 0.18 & $12.7^{\mathrm{b}}$ & 1.22 & $60.3^{\mathrm{a}}$ & 0.77 & $66.6^{\mathrm{a}}$ & 0.882 & 3.5 & 0.83 & $0.6^{\mathrm{b}}$ & 0.73 \\
\hline 12 months & 1980 & $83.2^{\mathrm{a}}$ & 0.49 & 46.8 & 1.91 & 5.9 & 0.85 & 0.0 & 0.18 & $17.9^{\mathrm{a}}$ & 1.22 & $38.5^{\mathrm{b}}$ & 0.77 & $46.4^{\mathrm{b}}$ & 0.882 & 4.6 & 0.83 & $1.4^{\mathrm{ab}}$ & 0.73 \\
\hline$P$ value & & \multicolumn{2}{|c|}{$0.00^{* * *}$} & \multicolumn{2}{|c|}{$0.12^{\mathrm{ns}}$} & \multicolumn{2}{|c|}{$0.38^{\mathrm{ns}}$} & \multicolumn{2}{|c|}{$0.21^{\mathrm{ns}}$} & \multicolumn{2}{|c|}{$0.00^{* *}$} & \multicolumn{2}{|c|}{$0.00^{* * *}$} & \multicolumn{2}{|c|}{$0.00^{* * *}$} & \multicolumn{2}{|c|}{$0.63^{\mathrm{ns}}$} & \multicolumn{2}{|c|}{$0.04^{*}$} \\
\hline Front- upper & 330 & 85.6 & & 42.5 & & 5.8 & & 0.0 & & 21.0 & & 47.78 & & 55.63 & & 4.5 & & 1.86 & \\
\hline Front-middle & 330 & 85.3 & & 39.6 & & 6.9 & & 0.6 & & 21.6 & & 47.58 & & 55.57 & & 5.4 & & 1.63 & \\
\hline Front- lower & 330 & 87.8 & & 50.1 & & 4.8 & & 0.0 & & 17.9 & & 47.78 & & 53.99 & & 7.5 & & 3.40 & \\
\hline Mean Front & 990 & 86.2 & 0.40 & 44.1 & 1.56 & 5.8 & 0.69 & 0.2 & 0.15 & 20.2 & 0.99 & 47.71 & 0.63 & 55.06 & 0.72 & 5.8 & 0.68 & 2.30 & 0.59 \\
\hline Back -upper & 330 & 85.7 & & 54.3 & & 6.8 & & 0.2 & & 12.9 & & 42.7 & & 49.6 & & 2.3 & & 1.5 & \\
\hline Back-middle & 330 & 86.9 & & 57.2 & & 8.2 & & 0.0 & & 11.3 & & 43.3 & & 49.5 & & 2.7 & & 1.8 & \\
\hline Back - lower & 330 & 88.1 & & 55.0 & & 7.0 & & 0.0 & & 16.3 & & 43.3 & & 48.9 & & 2.7 & & 0.8 & \\
\hline Mean Back & 990 & 86.9 & 0.40 & 55.5 & 1.56 & 7.4 & 0.69 & 0.1 & 0.15 & 13.5 & 0.99 & 43.1 & 0.63 & 49.3 & 0.72 & 2.55 & 0.68 & 1.4 & 0.59 \\
\hline $\mathrm{P}$ value & & \multicolumn{2}{|c|}{$0.26^{\mathrm{ns}}$} & \multicolumn{2}{|c|}{$0.00^{* * *}$} & \multicolumn{2}{|c|}{$0.15^{\mathrm{ns}}$} & \multicolumn{2}{|c|}{$0.52^{\mathrm{ns}}$} & 0.0 & & 0.00 & & 0.0 & & 0.0 & & 0.2 & \\
\hline General & & & & & & & & & & & & & & & & & & & \\
\hline Mean upper & 1980 & $85.6^{\mathrm{b}}$ & 0.49 & 48.4 & 1.91 & 6.3 & 0.85 & 0.1 & 0.18 & 17.0 & 1.22 & 45.3 & 0.77 & 52.6 & 0.88 & 3.4 & 0.83 & 1.7 & 0.73 \\
\hline Mean middle & 1980 & $86.1^{b}$ & 0.49 & 48.4 & 1.91 & 7.5 & 0.85 & 0.3 & 0.18 & 16.5 & 1.22 & 45.5 & 0.77 & 52.5 & 0.88 & 4.1 & 0.83 & 1.7 & 0.73 \\
\hline Mean lower & 1980 & $87.9^{\mathrm{a}}$ & 0.49 & 52.6 & 1.91 & 5.9 & 0.85 & 0.0 & 0.18 & 17.1 & 1.22 & 45.6 & 0.77 & 51.4 & 0.88 & 5.1 & 0.83 & 2.1 & 0.73 \\
\hline$P$ value & & 0.0 & & 0.2 & & & $1^{\mathrm{ns}}$ & 0.5 & & 0.9 & & 0.9 & & & & 0.3 & & 0.9 & \\
\hline Overall mean & 5940 & 86.5 & 0.82 & 49.8 & 1.83 & 6.59 & 0.51 & 0.14 & 0.11 & 16.8 & 1.25 & 45.4 & 2.64 & 52.2 & 2.62 & 4.18 & 0.60 & 1.83 & 0.47 \\
\hline
\end{tabular}

s.e - Standard error $\quad \mathrm{P}<0.05, \quad \mathrm{P}<0.01, \quad \mathrm{P}<0.001$, ns. Non significant - $\mathrm{P}>0.05$.

a,b,c - Within features and within columns differences between means with different superscripts are statistically significant $(\mathrm{P}<0.05)$. 
Table 2 The results of statistical analysis related to hatching features investigated in different egg location groups of 8-months old breeders

\begin{tabular}{|c|c|c|c|c|c|c|c|c|c|c|c|c|c|c|c|c|c|c|c|}
\hline \multirow[t]{2}{*}{ Egg location } & \multirow[t]{2}{*}{$\mathrm{n}$} & \multicolumn{2}{|c|}{$\begin{array}{l}\text { Fertility } \\
\text { rate } \\
(\%)\end{array}$} & \multicolumn{2}{|c|}{$\begin{array}{c}\text { Early-phase- } \\
\text { deaths } \\
(\%)\end{array}$} & \multicolumn{2}{|c|}{$\begin{array}{l}\text { Middle-phase- } \\
\text { deaths } \\
(\%)\end{array}$} & \multicolumn{2}{|c|}{$\begin{array}{c}\text { Late-phase- } \\
\text { deaths } \\
(\%)\end{array}$} & \multicolumn{2}{|c|}{$\begin{array}{c}\text { Shell adhesive } \\
\text { deaths } \\
(\%)\end{array}$} & \multicolumn{2}{|c|}{$\begin{array}{c}\text { Hatchability of } \\
\text { total eggs } \\
(\%)\end{array}$} & \multicolumn{2}{|c|}{$\begin{array}{c}\text { Hatchability of } \\
\text { fertile eggs } \\
(\%)\end{array}$} & \multicolumn{2}{|c|}{$\begin{array}{l}\text { Surplus poult } \\
\text { rate } \\
(\%)\end{array}$} & \multicolumn{2}{|c|}{$\begin{array}{l}\text { Anomaly } \\
\text { rate } \\
(\%)\end{array}$} \\
\hline & & mean & s.e. & mean & s.e. & mean & s.e. & mean & s.e. & mean & s.e. & mean & s.e. & mean & s.e. & mean & s.e. & mean & s.e. \\
\hline \multicolumn{20}{|l|}{ Front } \\
\hline upper & 330 & 85.5 & & 45.0 & & 6.9 & & 0.0 & & 22.8 & & 38.5 & & 45.1 & & 5.0 & & 3.6 & \\
\hline middle & 330 & 84.9 & & 36.2 & & 7.6 & & 0.0 & & 29.2 & & 40.9 & & 48.2 & & 3.6 & & 0.0 & \\
\hline lower & 330 & 87.0 & & 53.7 & & 4.2 & & 0.0 & & 19.8 & & 37.9 & & 43.6 & & 4.5 & & 5.8 & \\
\hline General & 990 & 85.7 & 0.63 & 45.0 & 5.03 & 6.2 & 1.04 & 0.00 & 0.00 & 23.9 & 2.77 & 39.1 & 0.93 & 45.0 & 1.38 & 4.4 & 0.42 & 3.1 & 1.69 \\
\hline \multicolumn{20}{|l|}{ Back } \\
\hline upper & 330 & 84.6 & & 54.8 & & 3.6 & & 0.0 & & 15.7 & & 36.7 & & 43.4 & & 4.3 & & 3.8 & \\
\hline middle & 330 & 86.1 & & 56.0 & & 6.5 & & 0.0 & & 14.5 & & 35.8 & & 41.6 & & 4.2 & & 5.4 & \\
\hline lower & 330 & 87.0 & & 51.7 & & 9.0 & & 0.0 & & 17.9 & & 34.9 & & 40.1 & & 4.9 & & 2.3 & \\
\hline General & 990 & 85.9 & 0.71 & 54.2 & 1.27 & 6.3 & 1.56 & 0.0 & 0.00 & 16.1 & 1.00 & 35.8 & 0.53 & 41.7 & 0.95 & 4.5 & 0.21 & 3.8 & 0.88 \\
\hline $\mathrm{P}$ value & & \multicolumn{2}{|c|}{$0.92^{\mathrm{ns}}$} & \multicolumn{2}{|c|}{$0.15^{\mathrm{ns}}$} & & $0.95^{\mathrm{ns}}$ & \multicolumn{2}{|c|}{$1.00^{\mathrm{ns}}$} & \multicolumn{2}{|c|}{$0.06^{\mathrm{ns}}$} & \multicolumn{2}{|c|}{$0.04^{*}$} & \multicolumn{2}{|c|}{$0.08^{\mathrm{ns}}$} & \multicolumn{2}{|c|}{$0.82^{\mathrm{ns}}$} & \multicolumn{2}{|c|}{$0.73^{\mathrm{ns}}$} \\
\hline \multicolumn{20}{|l|}{ Mean } \\
\hline upper & 660 & 85.0 & 0.46 & 49.9 & 4.92 & 5.2 & 1.66 & 0.0 & 0.00 & 19.2 & 3.51 & 37.6 & 0.91 & 44.2 & 0.83 & 4.6 & 0.33 & 3.70 & 0.11 \\
\hline middle & 660 & 85.5 & 0.61 & 46.1 & 9.89 & 7.0 & 0.53 & 0.0 & 0.00 & 21.8 & 7.35 & 38.3 & 2.58 & 44.9 & 3.33 & 3.9 & 0.33 & 2.7 & 2.69 \\
\hline lower & 660 & 87.0 & 0.00 & 52.7 & 0.95 & 6.6 & 2.39 & 0.0 & 0.00 & 18.9 & 0.94 & 36.4 & 1.52 & 41.8 & 1.74 & 4.7 & 0.17 & 4.1 & 1.74 \\
\hline $\mathrm{P}$ value & & \multicolumn{2}{|c|}{$0.15^{\mathrm{ns}}$} & \multicolumn{2}{|c|}{$1.00^{\mathrm{ns}}$} & & $0.65^{\mathrm{ns}}$ & \multicolumn{2}{|c|}{$1.00^{\mathrm{ns}}$} & \multicolumn{2}{|c|}{$1.00^{\mathrm{ns}}$} & \multicolumn{2}{|c|}{$0.65^{\mathrm{ns}}$} & \multicolumn{2}{|c|}{$0.65^{\mathrm{ns}}$} & \multicolumn{2}{|c|}{$0.18^{\mathrm{ns}}$} & \multicolumn{2}{|c|}{$0.87^{\mathrm{ns}}$} \\
\hline
\end{tabular}


Table 3 The results of statistical analysis related to hatching features investigated in different egg location groups of 10-months old breeders

\begin{tabular}{|c|c|c|c|c|c|c|c|c|c|c|c|c|c|c|c|c|c|c|c|}
\hline \multirow[t]{2}{*}{ Egg location } & \multirow[t]{2}{*}{$\mathrm{n}$} & \multicolumn{2}{|c|}{$\begin{array}{c}\text { Fertility } \\
\text { rate } \\
(\%)\end{array}$} & \multicolumn{2}{|c|}{$\begin{array}{c}\text { Early-phase- } \\
\text { deaths } \\
(\%)\end{array}$} & \multicolumn{2}{|c|}{$\begin{array}{c}\text { Middle-phase- } \\
\text { deaths } \\
(\%)\end{array}$} & \multicolumn{2}{|c|}{$\begin{array}{c}\text { Late-phase- } \\
\text { deaths } \\
(\%)\end{array}$} & \multicolumn{2}{|c|}{$\begin{array}{c}\text { Shell adhesive } \\
\text { deaths } \\
(\%)\end{array}$} & \multicolumn{2}{|c|}{$\begin{array}{c}\text { Hatchability of } \\
\text { total eggs } \\
(\%)\end{array}$} & \multicolumn{2}{|c|}{$\begin{array}{c}\text { Hatchability of } \\
\text { fertile eggs } \\
(\%)\end{array}$} & \multicolumn{2}{|c|}{$\begin{array}{c}\text { Surplus poult } \\
\text { rate } \\
(\%)\end{array}$} & \multicolumn{2}{|c|}{$\begin{array}{c}\text { Anomaly } \\
\text { Rate } \\
(\%)\end{array}$} \\
\hline & & mean & s.e. & mean & s.e. & mean & s.e. & mean & s.e. & mean & s.e. & mean & s.e. & mean & s.e. & mean & s.e. & mean & s.e. \\
\hline \multicolumn{20}{|l|}{ Front } \\
\hline upper & 330 & 87.9 & & 41.8 & & 5.4 & & 0.0 & & 16.4 & & 63.9 & & 72.8 & & 3.1 & & 1.4 & \\
\hline middle & 330 & 88.8 & & 42.7 & & 6.4 & & 1.8 & & 15.5 & & 60.0 & & 67.6 & & 7.5 & & 2.3 & \\
\hline lower & 330 & 92.4 & & 50.6 & & 5.6 & & 0.0 & & 15.7 & & 66.1 & & 71.5 & & 7.5 & & 0.0 & \\
\hline General & 990 & 89.7 & 1.39 & 45.0 & 2.78 & 5.8 & 0.28 & 0.6 & 0.61 & 15.9 & 0.27 & 63.3 & 1.78 & 70.6 & 1.56 & 6.1 & 1.47 & 1.2 & 0.66 \\
\hline \multicolumn{20}{|l|}{ Back } \\
\hline upper & 330 & 89.7 & & 55.2 & & 11.7 & & 0.7 & & 9.0 & & 55.2 & & 61.5 & & 1.0 & & 0.0 & \\
\hline middle & 330 & 93.0 & & 65.4 & & 11.0 & & 0.0 & & 6.6 & & 57.6 & & 61.9 & & 1.3 & & 0.0 & \\
\hline lower & 330 & 91.8 & & 61.7 & & 5.3 & & 0.0 & & 12.8 & & 59.1 & & 64.4 & & 0.7 & & 0.0 & \\
\hline General & 990 & 91.5 & 0.97 & 60.8 & 3.00 & 9.3 & 2.05 & 0.2 & 0.23 & 9.5 & 1.80 & 57.3 & 1.15 & 62.6 & 0.90 & 1.0 & 0.19 & 0.0 & 0.00 \\
\hline $\mathrm{P}$ value & & & $34^{\mathrm{ns}}$ & & $0.02^{*}$ & & $16^{\mathrm{ns}}$ & & $.59^{\mathrm{ns}}$ & & $.02^{*}$ & & $.05^{*}$ & & $.01^{*}$ & & $.03^{*}$ & & $4^{\mathrm{ns}}$ \\
\hline \multicolumn{20}{|l|}{ General } \\
\hline upper & 660 & 88.8 & 0.90 & 48.5 & 6.68 & 8.6 & 3.14 & 0.3 & 0.35 & 12.7 & 3.70 & 59.6 & 4.39 & 67.1 & 5.64 & 2.1 & 1.05 & 0.7 & 0.68 \\
\hline middle & 660 & 90.9 & 2.12 & 54.1 & 11.36 & 8.7 & 2.33 & 0.9 & 0.91 & 11.0 & 4.42 & 58.8 & 1.21 & 64.7 & 2.84 & 4.4 & 3.10 & 1.1 & 1.14 \\
\hline lower & 660 & 92.1 & 0.30 & 56.1 & 5.55 & 5.4 & 0.18 & 0.0 & 0.00 & 14.3 & 1.47 & 62.6 & 3.49 & 67.9 & 3.56 & 4.1 & 3.44 & 0.0 & 0.00 \\
\hline$P$ value & & & $37^{\text {ns }}$ & & $.65^{\mathrm{ns}}$ & & $37^{\mathrm{ns}}$ & & $.52^{\mathrm{ns}}$ & & $.65^{\mathrm{ns}}$ & & $.65^{\mathrm{ns}}$ & & $867^{\mathrm{ns}}$ & & $.87^{\mathrm{ns}}$ & & $2^{\mathrm{ns}}$ \\
\hline
\end{tabular}

s.e. - Standard error; $\quad{ }^{*} \mathrm{P}<0.05 ; \quad{ }^{\mathrm{ns}}$ Non significant $(\mathrm{P}>0.05)$. 
Table 4 The results of statistical analysis related to hatching features investigated in different egg location groups of 12-months old breeders

\begin{tabular}{|c|c|c|c|c|c|c|c|c|c|c|c|c|c|c|c|c|c|c|c|}
\hline \multirow[t]{2}{*}{ Egg location } & \multicolumn{3}{|c|}{$\begin{array}{l}\text { Fertility } \\
\text { rate } \\
(\%)\end{array}$} & \multicolumn{2}{|c|}{$\begin{array}{c}\text { Early-phase- } \\
\text { deaths } \\
(\%)\end{array}$} & \multicolumn{2}{|c|}{$\begin{array}{c}\text { Middle-phase- } \\
\text { deaths } \\
(\%)\end{array}$} & \multicolumn{2}{|c|}{$\begin{array}{c}\text { Late-phase- } \\
\text { deaths } \\
(\%)\end{array}$} & \multicolumn{2}{|c|}{$\begin{array}{c}\text { Shell adhesive } \\
\text { deaths } \\
(\%)\end{array}$} & \multicolumn{2}{|c|}{$\begin{array}{c}\text { Hatchability of } \\
\text { total eggs } \\
(\%)\end{array}$} & \multicolumn{2}{|c|}{$\begin{array}{c}\text { Hatchability of } \\
\text { fertile eggs } \\
(\%)\end{array}$} & \multicolumn{2}{|c|}{$\begin{array}{c}\text { Surplus poult } \\
\text { rate } \\
(\%)\end{array}$} & \multicolumn{2}{|c|}{$\begin{array}{c}\text { Anomaly } \\
\text { rate } \\
(\%)\end{array}$} \\
\hline & $\mathrm{n}$ & mean & s.e. & mean & s.e. & mean & s.e. & mean & s.e. & mean & s.e. & mean & s.e. & mean & s.e. & mean & s.e. & mean & s.e. \\
\hline \multicolumn{20}{|l|}{ Front } \\
\hline upper & 330 & 83.3 & & 40.6 & & 5.0 & & 0.0 & & 23.9 & & 40.9 & & 49.1 & & 5.5 & & 0.7 & \\
\hline middle & 330 & 82.1 & & 39.9 & & 6.7 & & 0.0 & & 20.2 & & 41.8 & & 50.9 & & 5.2 & & 2.6 & \\
\hline lower & 330 & 83.9 & & 46.2 & & 4.7 & & 0.0 & & 18.1 & & 39.4 & & 46.9 & & 10.5 & & 4.4 & \\
\hline General & 990 & 83.10 & 0.54 & 42.2 & 2.00 & 5.5 & 0.64 & 0.0 & 0.00 & 20.8 & 1.68 & 40.7 & 0.71 & 48.9 & 1.15 & 7.0 & 1.72 & 2.6 & 1.08 \\
\hline \multicolumn{20}{|l|}{ Back } \\
\hline upper & 330 & 82.7 & & 52.9 & & 5.3 & & 0.0 & & 14.1 & & 36.4 & & 44.0 & & 1.5 & & 0.8 & \\
\hline middle & 330 & 81.5 & & 50.0 & & 6.9 & & 0.0 & & 12.9 & & 36.7 & & 45.0 & & 2.6 & & 0.0 & \\
\hline lower & 330 & 85.5 & & 51.5 & & 6.9 & & 0.0 & & 18.1 & & 36.1 & & 42.2 & & 2.5 & & 0.0 & \\
\hline General & 990 & 83.2 & 1.17 & 51.5 & 0.84 & 6.4 & 0.52 & 0.0 & 0.00 & 15.0 & 1.59 & 36.4 & 0.18 & 43.7 & 0.81 & 2.2 & 0.36 & 0.3 & 0.27 \\
\hline $\mathrm{P}$ value & & & $94^{\mathrm{ns}}$ & & $0.01^{*}$ & & $.33^{\mathrm{ns}}$ & & $1.00^{\mathrm{ns}}$ & & $0.07^{\mathrm{ns}}$ & & $0.00^{* *}$ & & $.02^{*}$ & & $05^{\mathrm{ns}}$ & & $11^{\mathrm{ns}}$ \\
\hline \multicolumn{20}{|l|}{ General } \\
\hline upper & 660 & 83.0 & 0.30 & 46.7 & 6.18 & 5.2 & 0.17 & 0.0 & 0.00 & 19.0 & 4.91 & 38.6 & 2.27 & 46.5 & 2.57 & 3.5 & 2.00 & 0.7 & 0.07 \\
\hline middle & 660 & 81.8 & 0.30 & 44.9 & 5.06 & 6.8 & 0.10 & 0.0 & 0.00 & 16.6 & 3.68 & 39.2 & 2.58 & 48.0 & 2.97 & 3.9 & 1.28 & 1.3 & 1.32 \\
\hline lower & 660 & 84.7 & 0.76 & 48.8 & 2.64 & 5.8 & 1.09 & 0.0 & 0.00 & 18.1 & 0.00 & 37.7 & 1.67 & 44.6 & 2.37 & 6.5 & 3.99 & 2.2 & 2.2 \\
\hline$P$ value & & & $10^{\mathrm{ns}}$ & & $0.65^{\mathrm{ns}}$ & & $.37^{\mathrm{ns}}$ & & $1.00^{\mathrm{ns}}$ & & $0.87^{\mathrm{ns}}$ & & $0.57^{\mathrm{ns}}$ & & $57^{\mathrm{ns}}$ & & $87^{\mathrm{ns}}$ & & $96^{\mathrm{ns}}$ \\
\hline
\end{tabular}


When comparing the results between the 8- and 10-month flock ages, hatchability of all eggs set and of fertile eggs was lowest, and the rate of poults with abnormalities was highest in the 8-month group. However, no significant differences were observed between the 8-month and the 12-month group with respect to the features examined, except for fertility rate. These results therefore do not confirm earlier findings that hatchability is worst in the smallest eggs compared to larger eggs. Karaçanta et al. (1977) and Kaygisız et al. (1994) found that hatchability of all eggs set and of the fertile eggs was lower in $69.5 \mathrm{~g}$ Bronze turkey eggs than in $70-90 \mathrm{~g}$ eggs.

The hatchability of all eggs set and of fertile eggs was higher in the eggs placed in the front of the incubator in all age groups than at other positions. Also, early-phase-deaths were lower in the front than in the back. Although the difference in middle-phase-deaths was not significant between the front and back of the incubator, they were nevertheless numerically higher in the back compared to the front part of the incubator.

Shell adhesion death rate, null poult rate and abnormal poult rate were highest among eggs placed in the front of the incubator. The significant differences between results from the front and back of the incubator suggest that the internal conditions of the incubator might not be uniform. Mauldin \& Buhr (1995b) reported that the conditions inside incubators are not uniform. No significant differences were observed between the eggs placed in the upper, middle and lower parts of the incubator with respect to the examined features (Tables 1, 2, 3 and 4). This result is in accordance with the findings of Reinhart \& Hurnik (1984) and Elibol et al. (2000).

The weight of eggs from the 8-month group (average $67.4 \mathrm{~g}$ ) was lower than the ideal hatching egg weight reported for turkeys (Karaçanta et al., 1977; Erişir, 1999). For this reason it is recommended that eggs from breeders younger than 10 months should not be used for hatching to prevent the hatching of small poults.

\section{Conclusions}

It is concluded that if all eggs placed in the incubator have similar weights, a significant difference between the upper, middle, and lower parts of the incubator with respect to hatching results will not occur. However, the observed differences in hatching results between the front and back of the incubator used in this study lead to the conclusion that the conditions inside this incubator may not be uniform. Therefore, it is recommended that the design of incubators should be adjusted to ensure uniformity in air conditioning, temperature, circulation and humidity throughout the setter and hatcher.

Although these results were obtained from a specific incubator, the locations inside and all applications (temperature, humidity, shaking, etc.) were within accepted standards. Therefore these results would be applicable to incubators used in Turkey.

\section{Acknowledgements}

This study was supported by Mehmet Akif Ersoy University.

\section{References}

Brunson, C.C. \& Godfrey, G.F., 1953. The relationship of egg-shape, egg weight, specific gravity and 21 day incubation weight loss to hatchability of broad-breasted Bronze turkey eggs. Poult. Sci. 32, 846- 849.

Camc1, Ö. \& Sarıca, M., 1991. Breeding of intensive turkeys. J. Tigem 36, 5-9. (in Turkish, English abstract).

Deeming, D.C., 1996. Large eggs: An incubation challenge. Poultry International, December 1996.

Elibol, O., Türkoğlu, M. \& Erol, H., 2000. Effects of eggs weight and eggs location in incubation on hatchability results of different age broiler breeder flock. J. Poult. Res. 2, 17-24. (in Turkish, English abstract).

Erensayın, C., 2000. Scientific-Technic-Practical Poultry. Broiler breeding and hatchability. Vol.1. 2nd rev. ed. Nobel Publication, Ankara, Turkey (in Turkish, English abstract).

Erişir, Z., 1999. The effect of age, body weight and egg weight on hatchability in parent Bronz turkey. Ph.D. thesis, University of Firat, Turkey (in Turkish, English abstract).

French, N.A., 1994a. Effect of incubation temperature on the gross pathology of turkey embryos. Br. Poult. Sci. 35, 363-371. 
French, N.A., 1994b. Do incubation temperature requirements vary between eggs. Proc. 9th European Poultry Conference, Vol II, World Poultry Science Association. Glasgow, U.K.

French, N.A., 1997. Modelling incubation temperature: the effects of incubator design, embryonic development and egg size. Poult. Sci. 76, 124-133.

French, N.A., 2002. Managing the incubation environment in commercial hatcheries to meet the requirements of the embryo. Avian Poult .Biol. Rev. 13, 179-185.

Hagger, C.D., Steiger, S. \& Marqueral, C., 1986. Embryonic mortality in chicken eggs as influenced by egg weight and inbreeding. Poult. Sci. 65, 812-814.

Jerrold, H.Z., 1996. Biostatistical Analysis. 3rd rev. ed., United States of America, Prentice- Hall International Pres.

Karaçanta, A., Aybey, M., Koçak, Ç. \& Gönül, T., 1977. The effect of egg weight on hatchability in parent Bronz turkey. J. Agri. Fac. Ege Uni. 13, 133-137 (in Turkish, English abstract).

Kaygısız, A., 1993. Research on hatchability characteristics of Pekin ducks. Turkish J. Vet. Anim. Sci. 17, 205-208 (in Turkish, English abstract).

Kaygısız, A., Temur, C., Öğretmen, T. \& Demirel, M., 1994. The Effect of egg weight on hatchability in parent Bronz turkey. Turkish J. Vet. Anim. Sci. 18, 225-228 (in Turkish, English abstract).

Mauldin, J.M. \& Buhr, R.J., 1995a. What is really happening in your incubation? International Hatchery Practise 9, 19-22.

Mauldin, J.M. \& Buhr, R.J., 1995b. Temperature variation in incubation. Poult. Sci. 74 (Suppl.1), 204.

Ogunshile, G. \& Sparks, N., 1995. Effect of broiler egg weight on hatchability. Br. Poult. Sci. 36, 861-862.

Reinhart, B.S. \& Hurnik, G.I., 1984. Traits affecting the hatching performance of commercial chicken broiler eggs. Poult. Sci. 63, 240-245.

Reinhart, B.S. \& Moran, E.T., 1979. Incubation characteristics of eggs from older small White turkeys with emphasis on the effects due to egg weight. Poult. Sci. 58, 1599-1605.

Sotherland, P.R., Spotile, J.R. \& Paganelli, C.V., 1987. Avian eggs barriers to the exchange of heat and mass. J. Exp. Zool. (Suppl. 1), 81-86.

SPSS, 2002. SPSS for Windows 11.00. SPSS Inc. Chicago, USA.

Tullett, S.G., 1990. Science and the art of incubation. Poult. Sci. 69, 1-15.

Wilson, H.R., 1991. Interrelationships of egg size, chick size, posthatching growth and hatchability. Wrld Poult. Sci. 47, 5-20. 